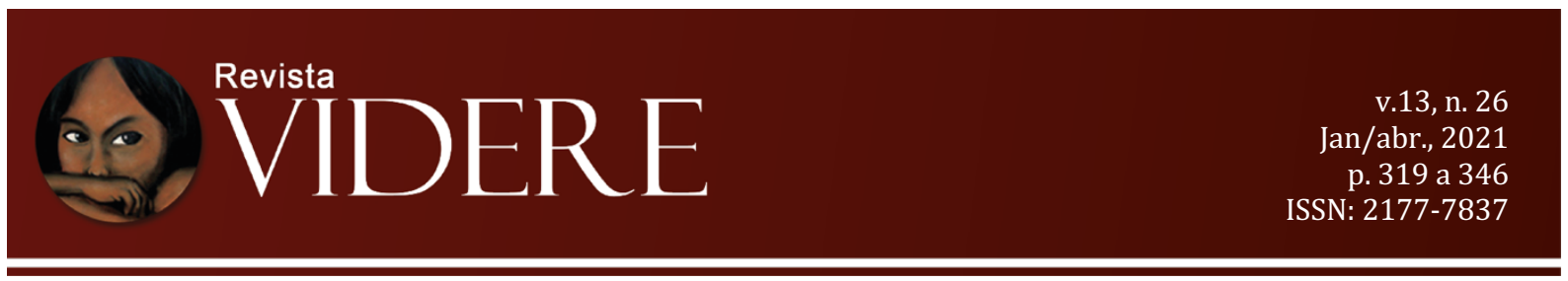

\title{
FRACKING, NEOEXTRACTIVISMO Y DERECHO HUMANO A LA SALUD
}

\author{
FRACKING NEO-EXTRACTIVISM AND THE HUMAN RIGHT TO HEALTH
}

FRACKING, NEOEXTRATIVISMO E O DIREITO HUMANO À SAÚDE

\begin{abstract}
Alberto Rojas Rueda
Maestro en políticas públicas, Facultad Latinoamericana de Ciencias Sociales, México. Candidato a doctor en derechos humanos, Universidad Autónoma de Tlaxcala/CNDH, México Coordinador de Desarrollo Profesional e Institucional del Instituto Mexicano de Tecnología del Agua E-mail: doctorrojas@,hotmail.com OrcidID: https://orcid.org/0000-0003-2959-2559
\end{abstract}

Resumen: El presente artículo aborda la relación entre la fractura hidráulica para extracción de hidrocarburos no convencionales, en adelante fracking, con el derecho humano a la salud, retomando conceptos teóricos del neoextractivismo, el metabolismo social y el enfoque de derechos humanos. A partir de una descripción de este derecho humano y sus dimensiones, así como de una revisión sistemática a la literatura científica relacionada con los efectos en salud del fracking, se identifican los impactos en salud de esta actividad en las personas y poblaciones que cohabitan con emprendimientos de fracking en sus territorios. El análisis de esta información verifica cómo estos impactos en salud derivan en violaciones a diferentes dimensiones del derecho humano a la salud que afectan mayormente a mujeres y a personas en condiciones de vulnerabilidad. Los hallazgos del trabajo permiten afirmar que las políticas de fomento del fracking, indirectamente promueven la violación de derechos humanos, en particular, a la salud. Esto se agrava cuando quienes sufren los impactos del fracking se encuentran sujetos a procesos estructurales de exclusión. El análisis aquí presentado fortalece los argumentos que proponen limitar o prohibir el fracking como medida para prevenir efectos en salud, limitar los procesos de exclusión para quienes cohabitan en el territorio con esta actividad neoextractiva y recomienda considerar el enfoque de derechos humanos como herramienta para la toma de decisiones gubernamentales en torno a actividades extractivas como el fracking.

Palabras clave: Fracking. neoextractivismo. salud. derechos humanos. 
Resumo: Este artigo aborda a relação entre o fraturamento hidráulico para a extração de hidrocarbonetos não convencionais, denominado fracking, com o direito humano à saúde, retomando conceitos teóricos do neoextrativismo, do metabolismo social e da abordagem dos direitos humanos. A partir da definição desse direito humano e de suas dimensões, bem como de uma revisão sistemática da literatura científica relacionada aos efeitos do fracking à saúde, são identificados os impactos dessa atividade na saúde de pessoas e populações que coabitam com empreendimentos de fracking. A análise dessas informações permite verificar como esses impactos na saúde resultam em violações de diferentes dimensões do direito humano à saúde, que afetam principalmente mulheres e pessoas em situação de vulnerabilidade. Os resultados do presente trabalho permitem afirmar que as políticas de fomento ao fracking promovem indiretamente a violação dos direitos humanos, em particular, à saúde. A gravidade da situação aumenta quando aqueles que sofrem os impactos do fracking estão sujeitos a processos estruturais de exclusão. A análise apresentada reforça os argumentos que propõem limitar ou proibir o fracking como uma medida para prevenir os efeitos à saúde e limitar os processos de exclusão para quem convive em ambientes com essa atividade neoextrativista, assim como recomenda considerar a abordagem dos direitos humanos como um instrumento para a tomada de decisões governamentais sobre atividades extrativas, como o fracking.

Palavras-chave: Fracking. neoextractivismo. saúde. direitos humanos.

Abstract: This article addresses the relationship between hydraulic fracturing for the extraction of unconventional hydrocarbons, hereinafter fracking, with the human right to health, taking up theoretical concepts of neo-extractivism, social metabolism, and human rights approach. Based on a description of this human right and its dimensions and a systematic review of the scientific literature, fracking's health impacts are described. The analysis of this information verifies how these health impacts lead to violations of different dimensions of the human right to health, mainly affecting women and vulnerable populations. The findings highlight that those policies promoting fracking indirectly promote human rights violations, particularly on health. This is more relevant when those who suffer the impacts of fracking are subject to systemic exclusion processes. To prevent fracking's negative health impacts, limit the systemic exclusion processes in their communities, this analysis supports the recommendation to limit or prohibit fracking. In addition, to suggest the human rights approach as a tool for decision-making processes around fracking policies.

Keywords: Fracking. neo-extractivism. health. human rights.

\section{Introducción}

La fractura hidráulica o también conocida como fracking es una tecnología utilizada para la extracción de hidrocarburos no convencionales contenidos en formaciones geológicas denominadas pizarras, lutitas o esquistos bituminosos (BJORLYKKE, 2015). Considerada una forma emergente de extractivismo, ha cobrado auge ante la disminución de los yacimientos convencionales de hidrocarburos, el incremento del precio del petróleo y la disponibilidad de innovaciones tecnológicas económicamente factibles (HILL, 2018). Los yacimientos que 
contienen estos hidrocarburos se encuentran distribuidas irregularmente en el planeta (ESTRADA, 2013). Estados Unidos de América (EUA), país dónde se desarrolló la tecnología, cuenta con importantes yacimientos de hidrocarburos en lutitas (PARRAGUEZ; UGARTE; CAMPERO, 2015) y ha invertido grandes recursos en su fomento, logrando un importante incremento de su producción (JANDA; KONDRATENCO, 2018). Países latinoamericanos como Argentina, Bolivia, Brasil, Chile, Colombia y México, cuentan con importantes yacimientos no convencionales de gran interés para la expansión del fracking en la región (ALIANZA LATINOAMERICANA FRENTE AL FRACKING, 2016).

La tecnología de fracking se introdujo "en la industria de la explotación de hidrocarburos en 1940 en Estados Unidos por la compañía Halliburton" (MANZANARES, 2014, p. 181).

El fracking, se puede utilizar para repotenciar yacimientos convencionales agotados, estimular pozos, o para extraer hidrocarburos de yacimientos no convencionales, Shale gas y Shale oil, por sus nombres en inglés (U. S. ENERGY INFORMATION ADMINISTRATION, 2020). En un yacimiento convencional a repotenciar, únicamente se realiza una perforación vertical, mientras que en un yacimiento no convencional se adiciona un pozo horizontal.

El fracking cobró relevancia en EUA a mediados de los años ochenta del siglo pasado (VARGAS; BARRIOS, 2013), con los emprendimientos en el Estado de Texas, particularmente en la cuenca Barnett (MANZANARES, 2014).

El proceso de fracking implica distintas fases: 1) Exploración/prospección de hidrocarburos (CHARRY-OCAMPO; PÉREZ, 2018); 2) Preparación del terreno e instalación de equipos para la mezcla y la recuperación de hidrocarburos; 3) Perforación de pozo, para el cuál primero se taladra verticalmente y posteriormente de forma horizontal. La perforación vertical es de entre uno y cinco kilómetros de profundidad (RAHM, 2011), en tanto la perforación horizontal tiene un "promedio de 1,600 metros" (MANZANARES, 2014); 4) La preparación de la mezcla a inyectar que se compone de "un fluido y un apuntalante" (U. S. ENERGY INFORMATION ADMINISTRATION, 2020). El fluido se compone de diversas sustancias, en su mayoría sintéticas y tóxicas (COLBORN, 2011) y abundantes cantidades de agua (WOOD, 2011) o en ocasiones gases como dióxido de carbono y nitrógeno (U. S. ENERGY INFORMATION ADMINISTRATION, 2020). En tanto el apuntalante se constituye principalmente de arenas silíceas "pero a veces se usa arena recubierta de resina, cerámica y bauxita sinterizada" (U. S. ENERGY INFORMATION, 2020). La mezcla generalmente se hace en el mismo sitio del emprendimiento; 5) La inyección mediante bombeo a "altas presiones, 
una mezcla de agua, arena y sustancias químicas, que romperán la roca impermeable y la mantendrán porosa" (AIDA-HEINRICH BÖLL STIFTUNG, 2016, p. 11), esta actividad se hace en ciclos que se repiten hasta agotar el yacimiento y es a la que se denomina fracturación hidráulica. (MANZANARES, 2014); 6) La recuperación del flujo de retorno (incluye gas, aceites, agua con mezcla de químicos y arenas); 7) La separación de los productos del flujo de retorno para su almacenamiento, transportación, reinyección o desecho; 8) El tratamiento y disposición de residuos; y, 9) El cierre (desmantelamiento y abandono de sitio).

A diferencia de los campos convencionales de hidrocarburos, el fracking implica una mayor intensidad de uso del territorio, tanto en extensión superficial como en el subsuelo, pues requiere perforar a mayor profundidad, más perforaciones (no solo verticales sino también horizontales), más cantidad de pozos, con un mayor uso de agua y energía, además de una importante excreción de emisiones contaminantes como gases de efecto invernadero, agua de retorno mezclada con sustancias tóxicas, entre otras (DE LA VEGA; RAMIREZ, 2015).

Las reservas de petróleo en tales depósitos exceden las reservas de petróleo convencionales, pero el alto costo y los problemas ambientales relacionados con la producción de este tipo de reservorios claramente limitan su explotación. Esto es particularmente cierto en la producción a partir de pizarra bituminosa (BJORLYKKE, 2015, p.4).

Cuando los pozos son abandonados, se generan diversos pasivos ambientales (CHARRY-OCAMPO; PÉREZ, 2018), que limitan retomar actividades productivas previamente existentes. El uso intensivo del territorio genera fuertes impactos negativos al ambiente, al clima global y a otras esferas del bienestar humano, lo que aunado a la colisión que tiene con otras actividades que se desarrollan en la superficie, es fuente de múltiples conflictos socio-ambientales (MARTINEZ-ALIER, 2002, 2009; NÚÑEZ, 2013).

Por otra parte, la independencia energética, los beneficios geoestratégicos que ofrece la producción de hidrocarburos a partir de yacimientos no convencionales, la idea de que el gas es un combustible fósil menos contaminante que otros hidrocarburos o el carbón para la generación de energía eléctrica, la generación de empleos y aumento del ingreso en las zonas dónde se realiza la actividad (HILL, 2018), refuerzan argumentos a favor del fracking, minimizando o nulificando, en el discurso, los riesgos que, para el ambiente, el clima o las personas pueda representar (BLACKWILL; O'SULLIVAN, 2014).

El fracking ha generado gran controversia. El flujo de información en medios de comunicación masiva y campañas promovidas por organizaciones de la sociedad civil sugieren que esta tecnología representa diversos riesgos para el bienestar humano. 
La literatura científica en torno a los impactos del fracking es utilizada por algunas comisiones o comités gubernamentales que requieren tomar la decisión de abrir o no sus territorios a esta tecnología. Algunos gobiernos han determinado prohibirla, otros establecen moratorias para su implementación (en tanto esperan a contar con mayor evidencia científica para determinar un curso de acción específico) y países como EUA (Colorado, Pensilvania, Texas) o Polonia, permiten y fomentan el fracking en sus territorios (NY DEPARTAMENT OF HEALTH, 2014; BJORLYKKE, 2015; ANGLÉS, 2016).

En este marco, se requiere un diálogo entre quienes deliberan en torno al futuro del fracking y quienes analizan, con evidencia, los impactos de actividades extractivas sobre los derechos humanos (DDHH) como se advierte de diversos estudios relacionados con el fracking y la salud (ROJAS-RUEDA, 2013), el ambiente (CARRY-OCAMPO; PÉREZ,, 2017), el derecho humano al agua, el derecho al territorio (FREIER; SHAJ, 2016) y sus afectaciones a pueblos indígenas (CARDOZA, 2017).

Sin embargo, de la revisión a la literatura, hasta el momento, no ha surgido un análisis que parta de un enfoque de DDHH para conocer, si el fracking representa un riesgo de violación a estos derechos fundamentales, así como los posibles alcances de estas violaciones. Avanzar en la construcción de conocimiento en torno a estos temas, podría coadyuvar en decisiones que, hasta el momento, no encuentran un cauce definido, al tiempo que permitirían retomar el foco de los DDHH como centro de las decisiones públicas en materia tanto de desarrollo como de política energética.

El presente trabajo presenta una ruta interdisciplinar que integra el enfoque de derechos humanos con el análisis de la literatura científica sobre los impactos en salud del fracking.

\section{Fracking como actividad neoextractiva}

La forma de relación humano-naturaleza, promovida por la civilización occidental, es la de dominación, que se verifica en el uso del concepto de recurso natural (en vez de elemento natural) el cual refleja una visión de apropiación humana de la naturaleza (LEFF, 2002), y facilita la normalización de las actividades extractivas (CANTE, 2015). Bajo este enfoque antropocéntrico hegemónico, se ha desarrollado la civilización.

Hasta las postrimerías del Siglo XVII, el consumo de recursos se gestó y desarrolló en relativa estabilidad con las capacidades regionales y globales de resistencia a estos impactos, manteniendo los sistemas naturales en condiciones óptimas para soportar el desarrollo civilizatorio (resiliencia del planeta a su antropización). 
A finales de ese mismo siglo, en la Europa Occidental, se desató el vertiginoso desarrollo industrial, que trajo como resultado un rápido agotamiento de los recursos naturales (BROSWIMMER, 2002), tanto renovables como no renovables y con ello, la necesidad de procurarse recursos naturales con técnicas más invasivas e intensas en esfuerzos energéticos, superficies de territorio ocupadas y cantidades de materiales movilizados (AZAMAR; PONCE, 2014). Esas técnicas extractivas, de mayor intensidad, concentradas en un producto específico o una categoría de recurso natural (peces, maderas, suelos para la agricultura o pastos para la ganadería, agua, minerales, combustibles), en el marco del modelo desarrollista industrialcivilizatorio de occidente, se denomina extractivismo.

"Los procesos de colonización de la naturaleza o actividades que alteran deliberadamente los sistemas naturales con el fin de hacerlos más útiles a la sociedad" (DELGADO, 2013, p. 10), fueron incrementados por la voracidad de los nuevos intereses de la clase burguesa industrial. La industrialización base del progreso civilizatorio, daría paso a una exacerbación de la colonización del hombre sobre espacios naturales, el incremento de conflictos por las tierras a colonizar, así como a muchas de las guerras del continente Euroasiático en siglos posteriores.

La nueva sociedad industrial para garantizar la operación de sus medios de producción, impuso primero la apropiación de la naturaleza y luego su extractivismo sobre otras formas posibles y preexistentes de relación con la naturaleza. Aparecieron las economías de enclave, impulsadas por procesos coloniales vigentes hasta nuestros días (FALERO, 2015 a). Esta visión colonialista potenció actividades como la trata de personas en busca de fuerza de trabajo para obtener al menor costo y con la mayor intensidad posible esos recursos naturales.

Gudynas (2016) habla del extractivismo de forma irónica como una construcción "teológica" (p. 13), pues se justifican en la creencia de que representa la única y mejor ruta para alcanzar el anhelado desarrollo civilizatorio, no en argumentaciones basadas en evidencia.

La nueva correlación entre el entorno natural y el mundo occidental moderno (industrializado), puede ser entendida como una forma de metabolismo (RIECHMANN, 2012), el metabolismo social o metabolismo socioeconómico (MARTÍNEZ-ALIER, 2009, 2010; TOLEDO, 2013; DELGADO, 2013). Gian Carlo Delgado (2013) lo define como aquel "uso diferenciado de insumos materiales, el procesamiento y los desechos de las sociedades, y la correspondiente producción energética" (p. 10), cuya estrategia de procuración de insumos para sus procesos (industriales) es el extractivismo, generando una serie de efectos conocidos e internalizados en sus dinámicas/procesos (agotamiento de insumos-recursos, consumo de energía, requerimiento de trabajo para extraer y procesar, acumulación de capital y bienes de 
producción, generación de desechos), y otros tantos que son desconocidos (alteración en ecosistemas, afectaciones a ciclos naturales, afectación al clima, impactos a la salud, alteraciones en las relaciones sociales y conflictos resultantes) y por tanto no son internalizados intencionalmente/racionalmente en sus procesos (externalidades) que terminan por impactar el metabolismo social (MARTINEZ-ALIER; KALLIS; VEUTHEY; WALTER; TEMPER, 2010).

La modalidad de la propiedad (comunal, social, pública, privada) y las dinámicas temporales (ya sea de corto o largo plazo) para la gestión de los recursos (DELGADO, 2013), determinaron en gran medida las externalidades negativas que ha provocado el modelo extractivista del desarrollo, muchas de las cuales, se han acumulado, siendo reconocidas e identificadas con mayor claridad hasta nuestros tiempos tales como el cambio climático, la sexta extinción masiva de especies, la caída global de las pesquerías, la desertificación, la contaminación, la violencia social, el incremento de enfermedades crónico-degenerativas, el incremento en la frecuencia de enfermedades emergentes, entre otras (PORRIT, 1991). Estas externalidades negativas, en la mayoría de los casos, implican distintos grados de violencia infringida por quienes promueven o realizan las actividades extractivas, sobre los que habitan o conviven en entornos extractivistas, quienes sufren en carne propia los impactos en salud, bienestar y paz, que desencadenan estas actividades, poniendo en "jaque la pervivencia de modos de uso de bienes naturales, y por ende modalidades de existencia social" (ASTELARRA; DE LA CAL; DOMÍNGUEZ, 2017, p. 236).

Así, el extractivismo es "un estilo de desarrollo basado en la apropiación de la naturaleza" (GUDYNAS, 2009, p. 188), en donde la tierra es vista como un medio de producción (acentuado por la propiedad privada) y, dónde el trabajo es considerado únicamente como un medio para extraer y transformar a gran escala los recursos naturales de dichos territorios (AZAMAR; PONCE, 2014).

Hablar de extractivismo lleva a imaginar emprendimientos mineros, energéticos, forestales, ganaderos, acuícolas, pesqueros o agrícolas, promovidas e impuestas desde centros de decisión sobre territorios con poblaciones que no son consultadas y consienten dichas actividades, que les generan efectos no deseados, ocasionando antagonismos que son fuente de conflictos, los denominados conflictos socioambientales (MARTINEZ-ALIER, 2002; TETREAULT; OCHOA; HERNÁNDEZ, 2012).

Los procesos extractivistas implican "violación de derechos y violencia contra los seres humanos que habitan en las comunidades afectadas. [Pues] Se «arranca» los recursos 
naturales imponiéndose con violencia, quebrándose el marco de derechos, y violándose los derechos humanos y de la naturaleza" (GROSFOGUEL, 2016, p. 126).

En este sentido, las actividades extractivas, por su naturaleza territorial, implican procesos de desterritorialización y reterritorialización (MURILLO; SACHER, 2017), que generan conflictos, conflictos socio-ambientales (MARTINEZ-ALIER, 2002), con distintos grados de violencia. La intensidad de la violencia asociada con el extractivismo se relaciona con "el acceso, despojo, uso y usufructo de territorios y los recursos ahí contenidos" (DELGADO, 2013, p. 9). "Este processo de despojo y usufructo privado de recursos no es algo nuevo sino algo estructural del sistema actual de producción com implicaciones socioambientales desiguales y sinérgicas" (DELGADO, 2013, p. 10).

Las concepciones epistémicas y ontológicas del extractivismo, nos llevan a pensar en la extrahección, esa forma de estar en el mundo violentando a los demás, normalizando la violencia y la violación sistemática de derechos humanos (GROSFOGUEL, 2016).

Delgado (2013) ha descrito, el modelo extractivista, como aquél que promueve “conflictos ecológicos distributivos" (p. 11), al interior de la sociedad, así como entre esta y su entorno natural.

En América Latina, al igual que en Europa, con el proceso de colonización europea, se rompieron las relaciones persona-comunidad-naturaleza que mantenían un vínculo de identidad e integralidad, más allá de la apropiación de espacios y recursos. Esta ruptura, ya sea por la eliminación total de la población preexistente ${ }^{1}$ o por su aculturación occidental (civilizatoria), promovió cambios drásticos en los entornos naturales y sociales del nuevo mundo (VÁZQUEZ; OROZCO, 1995).

Así, la ganadería y agricultura extensivas, la minería, la actividad forestal y las pesquerías a gran escala sustituyeron antiguas formas de relación y aprovechamiento de la naturaleza. El uso masivo de carbón, en gran medida promovido por la minería, y los desmontes para las actividades agropecuarias a mayor escala mermaron los bosques, modificando los usos de suelo y con ello empezó a generarse un incremento de contaminantes en las diferentes matrices (agua, suelo, aire, biodiversidad) en todo el continente americano.

Algunas comunidades originarias lograron aislarse (o fueron empujadas a un aislamiento forzoso) y con ello, resistir estos procesos, manteniendo sus formas de relación con la naturaleza, basados en la acción comunitaria y el reconocimiento de la biodiversidad como

\footnotetext{
1 "Ecocidio y etnocidio no son [...] sino las dos caras de una sola moneda: la de la implantación de un modelo de desarrollo basado en la acumulación y en la ganancia" (TOLEDO, 1989, p. 106).
} 
fuente de bienestar para la vida. Esta primera fase de extractivismo se vio potenciada con la industrialización acelerada en Europa y los EUA.

La sustitución de carbón por petróleo, como base de la producción y, por tanto, de la economía, provocó nuevos e importantes cambios en la relación con el entorno, pues los hidrocarburos se convertirían en el nuevo foco del interés de las industrias extractivas.

Así, una nueva etapa del extractivismo aparecería, con una mayor especialización y desarrollo tecnológico para potenciar las capacidades extractivas de la industria, lo que sumado a los nuevos y mejores medios de comunicación/transporte para las mercancías, llevaría de la mano del modelo capitalista, a agudizar los procesos extractivos, con el consecuente impacto sobre la naturaleza. La extinción de especies, la contaminación y la desertificación se agudizaron, de igual manera se terminaron de fundar los cimientos del cambio climático.

Una última ola de privatización de los escasos territorios en manos colectivas o públicas y un esfuerzo sin precedentes en la mercantilización de los recursos naturales (AZAMAR; PONCE, 2014), bajo el esquema del capitalismo neoliberal (auspiciado por el Estado), han provocado una aceleración de esta tendencia extractivista, dando paso a lo que Azamar y Ponce (2014) han denominado “neoextractivismo"(p. 139), un modelo económico basado en extracción abusiva de recursos naturales, promovido ahora no solo por el mercado sino por el Estado nacional mediante políticas económicas agresivas contra la naturaleza del mercado y a espaldas de las comunidades locales.

En toda América Latina, se han diseminado los extractivismos, entendidos estos como la apropiación de recursos naturales en grandes volúmenes o bajo prácticas de alta intensidad, para nutrir masivas exportaciones hacia la globalización. Los ejemplos más conocidos son la megaminería a cielo abierto, las perforaciones petroleras o las inmensidades de los monocultivos. Ese tipo de actividades se fortalecieron en especial a partir de inicios de la década de 2000, impulsados por altos precios y fuerte demanda (GUDYNAS, 2016).

En América Latina, el nuevo “estilo de desarrollo”, como lo llama Merchand (2016) fue brutalmente impuesto sobre las visiones locales de bienestar, en gran medida impulsadas por los modelos neoliberales, que promueven la explotación a gran escala de recursos naturales (reduciendo el capital natural de los países origen) y su exportación a países desarrollados (a precios que no internalizan todas las externalidades negativas de su extracción y movilización). Esta explotación no se limitó a la extracción de elementos naturales o a la pauperización del trabajo, sino que adoptó formas tan diversas como "la exposición a sustancias tóxicas" (TUNCAK, 2018, p. 1), derivadas de actividades neoextractivas. 
La relación de codependencia entre el Estado-mercado ${ }^{2}$, obliga a considerar las dinámicas políticas en los procesos que inciden sobre el modelo de desarrollo imperante y, por tanto, la forma como se relaciona, en estos tiempos, el ser humano con la naturaleza.

las relaciones de poder y los procesos de apropiación [se vinculan estrechamente] con el análisis de la producción, distribución y consumo propios de cada sistema de producción y de cara a los límites ambientales o la finitud de la naturaleza (DELGADO, 2013).

El modelo extractivista se finca en el discurso desarrollista neoliberal, interesado en obtener recursos naturales renovables (agua dulce, bosques, biodiversidad, pesca, suelos para la agricultura y la ganadería extensivas, luz solar y viento para las energías renovables) y no renovables (minerales y energéticos, principalmente hidrocarburos) a gran escala, manteniendo el modelo de concentración de riqueza, distribuyendo sus externalidades negativas de forma difusa y amplia (aunque a veces afecta principalmente ciertos segmentos de la población). Esta forma de desarrollo terminó por ampliar la brecha de la desigualdad e inequidad social y económica, provocando una ampliación y profundización de los daños ambientales y sociales concentrados en las zonas dónde se realizan las actividades. Además, promovió la desterritorialización por "ocupación y conversión de muchos territorios a la lógica del capital" (MURILLO; SACHER, 2017, p. 51), atentando contra la dignidad humana y por tanto contra los DDHH de las personas, generando descontento social que, en muchos casos, abreva la creciente conflictividad socioambiental que caracteriza al neoextractivismo latinoamericano.

Las actividades neoextractivas, parten de la lógica civilizatoria de apropiación de la naturaleza ${ }^{3}$, que rompe con visiones culturalmente aceptadas para muchos pueblos indígenas. Contiene distintos grados de violencia (contra las personas y la naturaleza) al no partir de un enfoque basado en DDHH (ORGANIZACIÓN MUNDIAL DE LA SALUD, 2017).

Las concesiones mineras o de hidrocarburos, sobre territorios poblados, en donde además se desarrollan otras actividades, distintas a la minería o a la exploración/extracción de hidrocarburos, chocan inevitablemente con los medios de vida previamente establecidos, haciendo visibles las colisiones de derechos y manifestándose como conflictos socioambientales (MARTINEZ-ALIER, 2009). La intensidad de estas colisiones de derechos, se exacerban en la medida en que se profundiza y hace más intensiva la explotación de los recursos naturales.

\footnotetext{
${ }^{2}$ Que se comporta como una captura política y económica del Estado por parte del mercado.

3 "El dominio sobre la naturaleza se convierte en elemento que define al ser humano como civilizado" (MIRÓN, 2005, p. 73).
} 
La industria petrolera y, en particular, los nuevos emprendimientos derivados de la extracción de hidrocarburos en yacimientos no convencionales, que demanda grandes territorios, energía, uso de grandes volúmenes de agua y otras materias primas (o sustancias sintéticas), impone restricciones y violenta diversos DDHH de las personas que habitan entornos extractivistas. La dignidad de los habitantes se pone en entredicho al imponerse un actor económico, sobre su bienestar. DDHH como la libre determinación de los pueblos, la seguridad e integridad física, el derecho a la cultura, a la salud, al ambiente sano, al agua, se afectan, lo que representa daño directo sobre las personas y refleja la violencia la actividad neoextractiva, muchas veces normalizada por nuestras sociedades.

En su mayoría, los capitales trasnacionales están desvinculados de los sitios dónde se obtienen sus beneficios económicos y de los impactos que genera su actividad (MERCHAND, 2016). Benefician a personas que se concentran en las grandes metrópolis y los países más ricos del planeta, que desconocen y se desentienden de quienes viven cerca de los yacimientos o las fuentes de los recursos naturales que los sostienen, un reflejo de la cara neocolonial del neoextractivismo.

Que las decisiones de un grupo violenten DDHH de otros sin mayor consecuencia para los primeros, normaliza la violencia transnacional, sostiene al neoextractivismo y lo subsume dentro del discurso desarrollista y de crecimiento económico.

La mayoría de las veces, los afectados ni siquiera tienen derecho a la consulta previa, libre e informada de las actividades económicas que les van a afectar. Concesiones, derechos mineros o para la exploración y extracción de elementos naturales, usos preferentes de sus territorios para transnacionales, todas promovidas por instituciones del Estado, quien se hace cómplice y coparticipe de la exportación de la naturaleza latinoamericana (MERCHAND, 2016). Esta violencia contra las comunidades locales se realiza de forma sistemática, simplemente por promover una política económica que incita actividades neoextractivas como el fracking ${ }^{4}$.

Así, la economía extractivista, se ha convertido en una fuente de conflicto socioambiental y en una suerte de violencia instituida por el modelo económico neoliberal hacia las personas más vulnerables: una violación sistemática y estructural de DDHH.

\section{EI derecho humano a la salud}

\footnotetext{
${ }^{4}$ Bajo esta perspectiva, el fracking representa una actividad neoextractivista, derivada de la fase neoliberal y su variante neo-desarrollista del capitalismo (ASTELARRA et al., 2017).
} 
Este derecho humano, es fundamental para la dignidad y realización plena de toda persona. Su desarrollo ha evolucionado desde una perspectiva originalmente de carácter médico-biológica hacia una más integral que incorpora aspectos socio ecológicos (PROSALUS; CRUZ ROJA ESPAÑOLA, 2014).

El derecho humano a la salud, de acuerdo con Prosalus y la Cruz Roja Española (2014) "debe entenderse como un derecho al disfrute de toda una gama de facilidades, bienes, servicios y condiciones necesarios para alcanzar el más alto nivel posible de salud" (p. 10).

El derecho humano a la salud se origina en la Constitución de la Organización Mundial de la Salud (OMS), en el marco de la Conferencia Internacional Sanitaria de 1946, previo a la Declaración Universal de los Derechos Humanos (DUDDHH), por lo que es un derecho humano internacionalmente reconocido previo a otros derechos inscritos en la DUDDHH.

La Constitución de la OMS define a la salud como: "Un estado completo de bienestar físico, mental y social, y no solamente la ausencia de afecciones de enfermedades" (ORGANIZACIÓN MUNDIAL DE LA SALUD, 2014).

En el artículo 25. 1 de la DUDDHH se establece que:

Toda persona tiene derecho a un nivel de vida adecuado que le asegure, así como a su familia, la salud y el bienestar, y en especial la alimentación, el vestido, la vivienda, la asistencia médica y los servicios sociales necesarios; tiene asimismo derecho a los seguros en caso de desempleo, enfermedad, invalidez, viudez, vejez y otros casos de pérdida de sus medios de subsistencia por circunstancias independientes de su voluntad (NACIONES UNIDAS, 1948).

Por su parte el Pacto Internacional de Derechos Económicos, Sociales y Culturales (PIDESC) adoptado en 1966, en su artículo 12 establece que:

1. Los Estados Partes en el presente Pacto reconocen el derecho de toda persona al disfrute del más alto nivel posible de salud física y mental.

2. Entre las medidas que deberán adoptar los Estados Partes en el Pacto a fin de asegurar la plena efectividad de este derecho, figurarán las necesarias para:

a) La reducción de la mortinatalidad y de la mortalidad infantil, y el sano desarrollo de los niños;

b) El mejoramiento en todos sus aspectos de la higiene del trabajo y del medio ambiente;

c) La prevención y el tratamiento de las enfermedades epidémicas, endémicas, profesionales y de otra índole, y la lucha contra ellas;

d) La creación de condiciones que aseguren a todos asistencia médica y servicios médicos en caso de enfermedad (NACIONES UNIDAS, 1966).

El Consejo Económico y Social de Naciones Unidas, a través del Comité de Derechos Económicos, Sociales y Culturales (CDESC) en su Observación General No. 14 del 
11 de agosto del año 2000, sobre cuestiones sustantivas relacionadas al disfrute del más alto nivel de salud que se plantean en la aplicación del PIDESC, dirigida a interpretar el artículo 12 del Pacto Internacional de Derechos Económicos, Sociales y Culturales. En sus artículos 1, 11, 15 y 16 reconoce a la salud como derecho humano fundamental, establece su interpretación, asociada a los determinantes en salud y al medio ambiente. De estos artículos se transcriben y algunos apartados considerados de importancia para el presente trabajo:

1. La salud es un derecho humano fundamental e indispensable para el ejercicio de los demás derechos humanos. Todo ser humano tiene derecho al disfrute del más alto nivel posible de salud que le permita vivir dignamente. 11. El Comité interpreta el derecho a la salud, definido en el apartado 1 del artículo 12, como un derecho inclusivo que no sólo abarca la atención de salud oportuna y apropiada sino también los principales factores determinantes de la salud, como el acceso al agua limpia potable y a condiciones sanitarias adecuadas, el suministro adecuado de alimentos sanos, una nutrición adecuada, una vivienda adecuada, condiciones sanas en el trabajo y el medio ambiente, y acceso a la educación e información sobre cuestiones relacionadas con la salud... Otro aspecto importante es la participación de la población en todo el proceso de adopción de decisiones sobre las cuestiones relacionadas con la salud en los planos comunitario, nacional e internacional.

15. El mejoramiento de todos los aspectos de la higiene ambiental e industrial (apartado b) del párrafo 2 del artículo 12) entraña, en particular, la adopción de medidas preventivas en lo que respecta a los accidentes laborales y enfermedades profesionales; la necesidad de velar por el suministro adecuado de agua limpia potable y la creación de condiciones sanitarias básicas; la prevención y reducción de la exposición de la población a sustancias nocivas tales como radiaciones y sustancias químicas nocivas $\mathrm{u}$ otros factores ambientales perjudiciales que afectan directa o indirectamente a la salud de los seres humanos. Además, la higiene industrial aspira a reducir al mínimo, en la medida en que ello sea razonablemente viable, las causas de los peligros para la salud resultantes del medio ambiente laboral. Además, el apartado b) del párrafo 2 del artículo 12 abarca la cuestión relativa a la vivienda adecuada y las condiciones de trabajo higiénicas y seguras...

16. La prevención y el tratamiento de las enfermedades epidémicas, endémicas, profesionales y de otra índole, y la lucha contra ellas (apartado c) del párrafo 2 del artículo 12) exigen que se establezcan programas de prevención y educación para hacer frente a las preocupaciones de salud que guardan relación con el comportamiento... y las que afectan de forma adversa a la salud sexual y genésica, y se promuevan los factores sociales determinantes de la buena salud, como la seguridad ambiental, la educación, el desarrollo económico y la igualdad de género (NACIONES UNIDAS, 2000).

De particular relevancia es el concepto de determinante en salud, que hace referencia a aquellas condiciones o situaciones que afectan la salud de las personas. Diversas actividades que se realizan en torno a emprendimientos neoextractivas, como el fracking, actúan como determinantes en salud. 


\section{El CDESC reconoce cuatro dimensiones esenciales e interrelacionadas del derecho} humano a la salud: disponibilidad, accesibilidad, aceptabilidad y calidad. A continuación, se transcriben los elementos que interesan para el presente trabajo.

a) Disponibilidad. Cada Estado Parte deberá contar con un número suficiente de... servicios públicos de salud... La naturaleza precisa de los servicios... dependerá de diversos factores, en particular el nivel de desarrollo del Estado Parte. Con todo, esos servicios incluirán los factores determinantes básicos de la salud, como agua limpia potable y condiciones sanitarias adecuadas...

b) Accesibilidad. Los establecimientos, bienes y servicios de salud deben ser accesibles a todos, sin discriminación alguna... La accesibilidad presenta cuatro dimensiones superpuestas:

i) No discriminación: los establecimientos, bienes y servicios de salud deben ser accesibles, de hecho y de derecho, a los sectores más vulnerables y marginados de la población, sin discriminación alguna por cualquiera de los motivos prohibidos.

ii) Accesibilidad física: los establecimientos, bienes y servicios de salud deberán estar al alcance geográfico de todos los sectores de la población, en especial los grupos vulnerables o marginados, como las minorías étnicas y poblaciones indígenas, las mujeres, los niños, los adolescentes, las personas mayores, las personas con discapacidades y las personas con VIH/SIDA. La accesibilidad también implica que los servicios médicos y los factores determinantes básicos de la salud, como el agua limpia potable y los servicios sanitarios adecuados, se encuentran a una distancia geográfica razonable, incluso en lo que se refiere a las zonas rurales. Además, la accesibilidad comprende el acceso adecuado a los edificios para las personas con discapacidades.

iii) Accesibilidad económica (asequibilidad): los establecimientos, bienes y servicios de salud deberán estar al alcance de todos. Los pagos por servicios de atención de la salud y servicios relacionados con los factores determinantes básicos de la salud deberán basarse en el principio de la equidad, a fin de asegurar que esos servicios, sean públicos o privados, estén al alcance de todos, incluidos los grupos socialmente desfavorecidos. La equidad exige que sobre los hogares más pobres no recaiga una carga desproporcionada, en lo que se refiere a los gastos de salud, en comparación con los hogares más ricos. iv) Acceso a la información: ese acceso comprende el derecho de solicitar, recibir y difundir información e ideas acerca de las cuestiones relacionadas con la salud...

c) Aceptabilidad. Todos los establecimientos, bienes y servicios de salud deberán ser respetuosos de la ética médica y culturalmente apropiados, es decir respetuosos de la cultura de las personas, las minorías, los pueblos y las comunidades, a la par que sensibles a los requisitos del género y el ciclo de vida...

d) Calidad. Además de aceptables desde el punto de vista cultural, los establecimientos, bienes y servicios de salud deberán ser también apropiados desde el punto de vista científico y médico y ser de buena calidad. Ello requiere..., agua limpia potable y condiciones sanitarias adecuadas (NACIONES UNIDAS, 2000).

A continuación, se presenta un cuadro con las dimensiones del derecho humano a

la salud. 


\begin{tabular}{|c|}
\hline Dimensiones del derecho humano a la salud \\
\hline Disponibilidad \\
\hline Accesibilidad \\
\hline Aceptabilidad \\
\hline Calidad \\
\hline
\end{tabular}

Cuadro 1- Dimensiones del derecho humano a la salud. Fuente: Elaboración propia.

\section{El fracking y el derecho humano a la salud}

La discusión en torno al fracking, se acompaña de elementos que sugieren una relación entre esta tecnología y la violación sistemática de DDHH. Para abordar la relación retomaré el concepto de determinante de salud.

Como se expuso en el primer capítulo, la tecnología del fracking tiene diversas fases de implementación, todas estas fases pueden relacionarse con afectaciones en salud. Mediante una revisión sistemática de la literatura científica internacional existente entre 1960 a 2017, y a la revisión realizada por Bamber et al. (2019), se identificaron artículos científicos que relacionan la exposición al fracking con algún tipo de efecto adverso sobre la salud, siendo los embarazos de alto riesgo, los partos prematuros (peso y talla bajos al nacimiento), los defectos congénitos del corazón y las exacerbaciones de asma, los que cuentan con evidencia suficiente para considerarlos como enfermedades asociadas a fracking (MCKENZIE et al, 2014; STACY et al, 2015; CASEY et al, 2016; RASMUSSEN et al, 2016; HILL, 2018; BAMBER et al., 2019).

Los trabajos analizados, se realizaron con poblaciones expuestas en los EUA, en condiciones en las que la industria cumple a cabalidad la normatividad de seguridad industrial y de protección al ambiente. Es menester considerar también, que EUA es un país en el que las medidas de control, inspección, vigilancia y sanción del marco normativo se consideran efectivas. Por ello, la correlación entre estas enfermedades y el fracking, se tienen, como una condición de la implementación de la tecnología, en las mejores condiciones de seguridad posibles, por lo que no existe argumento que pueda justificar que mayores medidas de seguridad industrial podrían evitar la aparición de estos padecimientos.

Con estos antecedentes, a la fecha, la única medida que garantizaría la aparición de estas enfermedades es la prevención de la exposición y dentro de esta medida la más efectiva es la prohibición de la actividad (tomando en cuenta la interdependencia de DDHH como se describirá más adelante). 
Tres de las enfermedades, embarazos de alto riesgo, parto prematuro y defectos congénitos del corazón tienen implicaciones especiales, por la implicación de mujeres y niños entre sus potenciales afectados. Así, la Observación General 14 del CDESC en sus Artículos 12 - Temas especiales de alcance general, 21 -La mujer y el derecho a la salud, y 22 -Los niños y adolescentes, se refieren explícitamente a luchar contra la discriminación contra la mujer y los niños promoviendo medidas que garanticen entornos seguros, su salud a lo largo de su vida, incluyendo medidas preventivas que ataquen los riesgos que afectan a su salud asociadas con mortalidad materna, así como la mortalidad y morbilidad prenatal. Se transcriben los artículos 21 y 22:

La mujer y el derecho a la salud

21. Para suprimir la discriminación contra la mujer es preciso elaborar y aplicar una amplia estrategia nacional con miras a la promoción del derecho a la salud de la mujer a lo largo de toda su vida. Esa estrategia debe prever en particular las intervenciones con miras a la prevención y el tratamiento de las enfermedades que afectan a la mujer, así como políticas encaminadas a proporcionar a la mujer acceso a una gama completa de atenciones de la salud de alta calidad y al alcance de ella, incluidos los servicios en materia sexual y reproductiva. Un objetivo importante deberá consistir en la reducción de los riesgos que afectan a la salud de la mujer, en particular la reducción de las tasas de mortalidad materna y la protección de la mujer contra la violencia en el hogar. El ejercicio del derecho de la mujer a la salud requiere que se supriman todas las barreras que se oponen al acceso de la mujer a los servicios de salud, educación e información, en particular en la esfera de la salud sexual y reproductiva. También es importante adoptar medidas preventivas, promocionales y correctivas para proteger a la mujer contra las prácticas y normas culturales tradicionales perniciosas que le deniegan sus derechos genésicos.

Los niños y adolescentes

22. En el apartado a) del párrafo 2 del artículo 12 se pone de manifiesto la necesidad de adoptar medidas para reducir la mortinatalidad y la mortalidad infantil y promover el sano desarrollo de los niños. En los ulteriores instrumentos internacionales de derechos humanos se reconoce que los niños y los adolescentes tienen derecho al disfrute del más alto nivel posible de salud y al acceso a centros de tratamiento de enfermedades. En la Convención sobre los Derechos del Niño se exhorta a los Estados a que garanticen el acceso a los servicios esenciales de salud para el niño y su familia, incluida la atención anterior y posterior al parto de la madre. La Convención vincula esos objetivos con el acceso a la información, respetuosa del niño, sobre prevención y fomento de la salud y la prestación de ayuda a las familias y comunidades para poner en práctica esas medidas. La aplicación del principio de no discriminación requiere que tanto las niñas como los niños tengan igual acceso a una alimentación adecuada, un entorno seguro y servicios de salud física y mental (NACIONES UNIDAS, 2000).

Los partos prematuros embarazos de alto riesgo ponen en peligro la vida de la madre y el embrión/nonato, lo que implica que el Estado no cumpliría la protección del derecho humano a la salud en estos segmentos de la población, en particular emitiendo medidas que las 
protejan contra agentes estatales o no estatales que promueven y realizan fracking. Esto, a pesar de que lo hagan en el marco de la ley, pues, como la evidencia científica lo demuestra, no existe condición de seguridad para la realización de esta actividad. Bajo estas consideraciones, el Estado debería adoptar medidas inmediatas para prevenir esta condición y con ello, obligarse a respetar, proteger y cumplir el derecho humano a la salud de las mujeres.

La misma suerte tiene la relación del fracking con defectos congénitos del corazón, dónde se pone en riesgo la vida del embrión, del neonato y de los niños, puede condicionar su vida, salud y desarrollo futuro. La falta de un entorno seguro, que debe garantizar el Estado, para el desarrollo intrauterino durante el embarazo, condiciona esta situación. El Estado habiendo conocido el riesgo de violación de derecho humano a la salud a los niños está obligado a imponer medidas para proteger la salud materno-fetal.

En este sentido, el Relator Especial sobre las Obligaciones de Derechos Humanos Relacionadas con la Gestión y Eliminación Ecológicamente Racionales de las Sustancias y los Residuos Peligrosos en su reporte especial presentado en la 73 Asamblea General de las Naciones Unidas afirmó que "la exposición a la contaminación tóxica es la principal fuente de muerte prematura en el mundo en desarrollo" (TUNCAK, 2018).

Los pediatras describen a los niños de hoy como nacidos precontaminados por un cóctel de sustancias incuestionablemente tóxicas, muchas de las cuales no tienen un nivel seguro de exposición o que años después se demostró que eran más tóxicas de lo que se suponía inicialmente. Una pandemia silenciosa de enfermedad, discapacidad y muerte prematura está ahora muy extendida, en gran parte debido a la exposición infantil durante los períodos sensibles del desarrollo (TUNCAK, 2018).

La exacerbación de crisis asmáticas, se ciñe sobre población asmática, lo que hace que la relación fracking derecho humano a la salud se cebe sobre la población más vulnerable (mujeres, niños, niños con asma, adultos mayores con asma).

De lo anterior, se deriva que existe evidencia científica suficiente que correlaciona al fracking con afectaciones a la salud, pese a que se cumpla a cabalidad con toda la regulación de seguridad industrial y ambiental preexistente.

De realizarse el fracking, independientemente de las medidas de prevención que se impongan, la afectación a la salud se presentará, en particular en mujeres en edad reproductiva principalmente en embarazadas, también en nonatos, recién nacidos y personas asmáticas.

Si consideramos que estas actividades se realizan principalmente en zonas rurales, la vulnerabilidad estructural se agudiza. A esta vulnerabilidad, se debe sumar que las mujeres "viven en situación de desigualdad social, política y económica generalizada" (OLIVERA, 2019, p. 9). La evidencia científica en materia de salud indica que el fracking, afecta de manera 
diferenciada a hombres y mujeres, siendo las mujeres más susceptibles a afectaciones en salud. Esta situación incrementa la discriminación sobre las mujeres lo que además contraviene la Convención sobre la Eliminación de todas las formas de Discriminación contra la Mujer de las Naciones Unidas (NACIONES UNIDAS, 1979).

Cabría la posibilidad de un escenario aún más adverso, en el que se despliega el fracking en condiciones de regulación industrial o ambiental laxas, insuficientes o teniendo esta regulación bien desarrollada, pero con capacidades limitadas de control (inspección, vigilancia y sanción) efectivos por parte del Estado. En cualquiera de estas condiciones, se esperaría que los impactos en salud sean mayores a los reportados en la literatura y, por tanto, la afectación a grupos en condiciones de vulnerabilidad sería más severa. Si a estos escenarios se agrega que se realice la actividad sobre territorios con población en pobreza, excluida o estructuralmente discriminada, una mayor afectación a la salud es previsible.

Esta hipotética situación podemos verla en el caso mexicano. De acuerdo con Olivera (2019), el fracking afectaría el territorio de 6,664 localidades de menos de 2,500 habitantes (rurales), en 118 municipalidades de siete estados de la República Mexicana. Con una población total de 4,106,091 de habitantes, de los cuáles $51 \%$ son mujeres y $49 \%$ son hombres. En las localidades potencialmente afectadas, el $73 \%$ de las mujeres se encuentra en el rango entre 15 y 49 años de edad (que abarca la edad reproductiva); 26\% no están afiliadas a alguna institución de salud pública; más de 380,000 no tienen acceso a agua potable; 57,595 son analfabetas; solo $8 \%$ reportan un empleo formal, y del total de la población indígena registrada, 134, 237, que representa el 48\%, son mujeres. Estas condiciones adversas y de exclusión estructural, en un país como México, evidencian el riesgo de afectaciones a la salud para las mujeres que representaría el fracking.

Considerando que el Estado tiene la obligación de promover, respetar, proteger y cumplir ${ }^{5}$ los $\mathrm{DDHH}$, sin distinción y que el derecho a la salud es un derecho humano inalienable, con la evidencia científica disponible, que reconoce la relación entre el fracking y afectaciones a la salud, los Estados firmantes de alguno de los instrumentos internacionales en materia de DDHH aquí descritos, están obligados a prevenir violaciones a los DDHH derivadas de la implementación de esta tecnología.

Para los casos en los que se ha evidenciado impactos en salud, el fracking representa hoy en día una violación flagrante a DDHH, en particular al derecho humano a la salud. De

\footnotetext{
${ }^{5}$ En tratados internacionales y derecho consuetudinario los estados tienen tres deberes con los DDHH: respetar, proteger y cumplir. (UNIÓN INTERPARLAMENTARIA, 2016, p. 33)
} 
continuarse la afectación para estas comunidades (en EUA), habría una violación continuada y sistemática a DDHH, lo que podría considerarse una violación grave. En dicho contexto, por lo que corresponde a derecho humano a la salud, cualquier Estado que promueva el fracking, ejecuta una acción contraria al principio de no discriminación en su relación con la salud.

No se debe olvidar que los Estados tienen obligación de proteger mediante la adopción de leyes u otras medidas a "grupos vulnerables o marginados de la sociedad, en particular las mujeres, los niños, los adolescentes y las personas mayores, teniendo en cuenta los actos de violencia desde la perspectiva de género" (NACIONES UNIDAS, 2000, p. 10) El fracking acrecienta la carga de problemas sanitarios en grupos vulnerables y marginados que ya soportan una proporción excesiva de estos en la sociedad (ORGANIZACIÓN MUNDIAL DE LA SALUD, 2017). Limitar la información relacionada con impactos en salud del fracking, o desvirtuar intencionalmente información respecto de sus impactos en salud violenta este principio. "Los Estados deben velar asimismo porque terceros no limiten el acceso de las personas a la información” (NACIONES UNIDAS, 2000, p. 10).

La recomendación 34 de la Observación General al Artículo14 del CDESC establece que "los Estados deben abstenerse asimismo de contaminar ilegalmente la atmósfera, el agua y la tierra, por ejemplo, mediante los desechos industriales de las instalaciones propiedad del Estado..." (NACIONES UNIDAS, 2000, p. 10), condición que no se observa, en su relación con el principio de respetar el derecho humano a la salud asociado con el fracking.

El fracking afecta los determinantes en salud relacionados con las cuatro dimensiones del derecho humano a la salud, independientemente del lugar dónde se encuentre.

En cuanto a la dimensión de accesibilidad, tres de los subcomponentes de esta dimensión: accesibilidad física, económica y de información, están directamente relacionados con el fracking (agua y condiciones sanitarias en el entorno).

Existen otras situaciones asociadas con el derecho humano a la salud, que deben ser revisadas con mayor profundidad. Tal es el caso de su relación con pueblos indígenas. Se tiene conocimiento que la actividad petrolera ha generado alteración en el entorno natural, la relación de los pueblos con su medio y desplazamiento forzado de poblaciones indígenas en diversas partes del mundo, en particular en América Latina. En México, esta situación se ha verificado en la zona del Totonacapan (Vertiente del Golfo de México), dónde se han presionado recursos naturales de pueblos originarios causando problemas diversos, muchas veces terminando en desplazamiento forzado de comunidades (OLIVERA, 2019). La Observación General 14 (2000) del CDESC, también considera una particularidad en relación a pueblos indígenas. En este sentido, reconoce que actividades promovidas por ciertos modelos 
de desarrollo pueden ejercen un efecto perjudicial sobre a salud de las poblaciones indígenas y ello es una verificación de violación al derecho humano a la salud.

\section{Pueblos indígenas}

27. Habida cuenta del derecho y la práctica internacionales que están surgiendo, así como de las medidas adoptadas recientemente por los Estados en relación con las poblaciones indígenas, el Comité estima conveniente identificar los elementos que contribuirían a definir el derecho a la salud de los pueblos indígenas, a fin de que los Estados con poblaciones indígenas puedan aplicar más adecuadamente las disposiciones contenidas en el artículo 12 del Pacto. El Comité considera que los pueblos indígenas tienen derecho a medidas específicas que les permitan mejorar su acceso a los servicios de salud y a las atenciones de la salud. Los servicios de salud deben ser apropiados desde el punto de vista cultural, es decir, tener en cuenta los cuidados preventivos, las prácticas curativas y las medicinas tradicionales. Los Estados deben proporcionar recursos para que los pueblos indígenas establezcan, organicen y controlen esos servicios de suerte que puedan disfrutar del más alto nivel posible de salud física y mental. También deberán protegerse las plantas medicinales, los animales y los minerales que resultan necesarios para el pleno disfrute de la salud de los pueblos indígenas (NACIONES UNIDAS, 2000).

El Comité observa que, en las comunidades indígenas, la salud del individuo se suele vincular con la salud de la sociedad en su conjunto y presenta una dimensión colectiva. Al respecto, el Comité considera que las actividades que inducen al desplazamiento de poblaciones indígenas, contra su voluntad, de sus territorios y entornos tradicionales, con la consiguiente pérdida de sus recursos alimenticios y la ruptura de su relación simbiótica con la tierra, ejercen un efecto perjudicial sobre a salud de esas poblaciones.

El Convenio 169 sobre Pueblos Indígenas y Tribales, de la Organización Internacional del Trabajo, obliga a los gobiernos a proteger los derechos de los pueblos indígenas, garantizar su integridad y a no emplear ninguna forma de coerción que viole sus derechos humanos (ORGANIZACIÓN INTERNACIONAL DEL TRABAJO, 1989).

\subsection{Violación del derecho humano a la salud}

Existe violación de DDHH cuando "el Estado incumple sus obligaciones de garantizar que los derechos económicos, sociales y culturales se disfruten sin discriminación, o su obligación de respetarlos, protegerlos y realizarlos" (CONSEJO NACIONAL DE EVALUACIÓN DE LA POLÍTICA DE DESARROLLO SOCIAL, 2018, p. 23).

La Observación General 14(2000) del CDESC, en su Apartado III. Violaciones, reconoce que cuando no existe una incapacidad estructural del Estado para atender el cumplimiento del derecho humano a la salud, y éste, cae en acciones u omisiones que violan 
este derecho, se incumple con sus obligaciones internacionalmente contraídas (NACIONES UNIDAS, 2000, p. 14)

Por tanto, para determinar posibles violaciones a DDHH, es necesario correlacionar estas acciones con las obligaciones del Estado. A continuación, se analizan cada una de las obligaciones en su relación con el fracking.

En lo que respecta a la obligación de respetar que implica "las acciones, políticas o leyes de los Estados que contravienen las normas establecidas en el artículo 12 del Pacto y que son susceptibles de producir lesiones corporales, una morbosidad innecesaria y una mortalidad evitable" (NACIONES UNIDAS, 2000, p. 14), así como "la suspensión de la legislación o la promulgación de leyes o adopción de políticas que afectan desfavorablemente al disfrute de cualquiera de los componentes del derecho a la salud" (NACIONES UNIDAS, 2000, p. 14)

La promoción del fracking desde la legislación, así como la implementación de políticas y programas que buscan incrementar la producción de hidrocarburos utilizando esta tecnología es contraria esta obligación. También se ve violentada con "la ocultación o tergiversación deliberadas de la información que reviste importancia fundamental para la protección de la salud" (NACIONES UNIDAS, 2000, p. 14). Existe evidencia científica sobre dichas afectaciones, al menos en lo que corresponde al derecho humano a la salud, por lo que, promover esta actividad en cualquier parte del mundo, ocultando o tergiversando información para prevenir sus efectos en salud, es una violación a la obligación de respetar dicho derecho humano.

La misma Observación General 14 (2000) del CDESC considera como una violación a proteger el derecho humano a la salud, cuando "un Estado no adopte todas las medidas necesarias para proteger, dentro de su jurisdicción, a las personas contra las violaciones del derecho a la salud por terceros. Figuran en esta categoría omisiones tales como la no regulación de las actividades de particulares, grupos o empresas con objeto de impedir que esos particulares, grupos o empresas violen el derecho a la salud de los demás" (NACIONES UNIDAS, 2000, p.15), como ocurre en el caso de la implementación del fracking promovida o facilitada por el Estado en cualquier territorio. Y en relación con industrias extractivas, como la de hidrocarburos, la Observación 14(2000), también considera como violación a la obligación de proteger "el no promulgar o hacer cumplir las leyes a fin de impedir la contaminación del agua, el aire y el suelo por las industrias extractivas" (NACIONES UNIDAS, 2000, p. 15), como es el caso de la legislación y regulación que autoriza, permite, fomenta o promueve el fracking, pese a los impactos en salud conocidos. 
En lo que respecta a cumplir, se actualiza "cuando los Estados Partes no adoptan todas las medidas necesarias para dar efectividad al derecho a la salud" (NACIONES UNIDAS, 2000, p. 15). Existen cuatro componentes que la Observación General 14 (2000), reconoce como violaciones a la obligación de cumplir que pueden relacionarse directamente con el fracking:

Los gastos insuficientes o la asignación inadecuada de recursos públicos que impiden el disfrute del derecho a la salud; La no vigilancia del ejercicio del derecho a la salud en el plano nacional; La no adopción de un enfoque de la salud basado en la perspectiva de género; y El hecho de no reducir las tasas de mortalidad infantil y materna (NACIONES UNIDAS, 2000, p. 15).

Por lo anterior y derivado de lo expuesto en el apartado que precede, se verifica que cuando un Estado promueve o facilita el fracking violenta el derecho humano a la salud. Esta violación puede ser continuada y agravar las condiciones estructurales de discriminación y exclusión de los más desfavorecidos. Por dichas razones el fracking debería considerarse una violación grave de DDHH.

Bajo esta consideración, es obligación de los Estados evitar y, en su caso, detener la continuación del fracking, proteger a las víctimas, promover la reparación del daño a las personas afectadas y prevenir futuras violaciones.

\section{Consideraciones finales}

El trabajo aporta elementos suficientes para reconocer que el fracking violenta el derecho humano a la salud de las personas que habitan los territorios dónde se desarrolla. Ello exhibe la necesidad de que los Estados actúen y garanticen los DDHH afectados por el fracking.

Ante este escenario, considerando por una parte las condiciones estructurales de discriminación, marginación y exclusión de ciertos grupos de la población; la debilidad institucional de los países latinoamericanos en lo relativo a la regulación ambiental y a su control; y por otra, las recomendaciones del relator especial sobre residuos y sustancias tóxicas, que equiparan la liberación de sustancias tóxicas al ambiente con formas de violencia similares a las derivadas de luchas armadas, que permite equiparar al fracking con la violencia armada. El fracking desde un enfoque de DDHH es una actividad que implica un alto nivel de violencia hacia las personas, sus colectivos y al ambiente.

En una sociedad enmarcada en la búsqueda de un desarrollo con equidad, integral y sustentable, no cabe la posibilidad de aceptar, tolerar o normalizar la violencia contra las personas, las comunidades o el ambiente, pues, ello no genera condiciones de paz, necesarias 
para lograr la dignidad de las personas. El fracking, un desarrollo tecnológico que en sus orígenes fue pensado para extraer fuentes de energía basada en hidrocarburos difícilmente aprovechables, no consideró (como la mayoría de los desarrollos tecnológicos) la integralidad de los efectos que podría generar en las personas, la sociedad y el ambiente.

La perspectiva de DDHH, coloca al fracking, como una actividad contraria a los deseos y las aspiraciones de la sociedad mundial, plasmados en la mayoría de los documentos multilaterales en materia de DDHH firmados en el marco del Sistema de las Naciones Unidas y de otros Sistemas Regionales. No cabe duda, que mantener, facilitar y promover esta actividad, activa o pasivamente, representa una afrenta contra este marco de actuación multinacional y en contra del modelo de desarrollo promovido desde las Naciones Unidas con la Agenda 2030. Se puede afirmar con evidencia, en materia de salud, que el fracking, va en contra del bienestar de las personas, al atentar contra su dignidad.

Adoptar esta tecnología bajo el argumento el derecho humano al desarrollo, es un contrasentido, pues desconoce la integralidad, interdependencia y progresividad de los DDHH que implican que, en ningún caso, se puede vulnerar un derecho humano para garantizar otro.

El fracking es un claro ejemplo de tecnología no acoplada a DDHH. Si bien, el desarrollo de esta tecnología buscó una alternativa para mantener el modelo de desarrollo basado en los hidrocarburos, demuestra la falta de integración de una perspectiva ética que incorpore los DDHH desde la concepción del desarrollo tecnológico hasta su implementación.

Acoplar la perspectiva ética a la innovación científica y tecnológica, con un enfoque de DDHH es un reto más de las presentes y futuras generaciones. La crisis ambiental global, aunada a la de DDHH, no dará tregua a seguir generando innovaciones tecnológicas y científicas que violenten DDHH en su implementación o en el proceso de su desarrollo. Los errores del pasado científico y tecnológico, deben servir para el aprendizaje y no repetirlos para bien de la humanidad. Lo que pasa con el fracking es un crimen las personas expuestas a sus efectos nocivos, algo que no podemos darnos el lujo de continuar, ni repetir.

\section{Referencias}

AIDA- Heinrich Böll Stiftung, Principio de Precaución: Herramienta jurídica ante los impactos del fracking. México: AIDA- Heinrich Böll Stiftung, México, Centro américa y el Caribe. 2016.

ALIANZA LATINOAMERICANA FRENTE AL FRACKING. Última Frontera: Políticas Públicas, impactos y resistencias al fracking en América Latina. Santiago: Fundación Heinrich Böll Cono Sur y Amigos de la Tierra Europa, 2016. 
ANGLÉS, Marisol. Fracturación hidráulica y su impacto en los derechos humanos a un medioambiente sano, al agua y a la salud. En: ACKERMAN, John. (coord.).

Fracking: ¿Qué es y cómo evitar que acabe con México? México: UNAM, Instituto de Investigaciones Jurídicas, 2016, pp. 31-57.

AZAMAR, Aleida; PONCE, José Ignacio. Extractivismo y desarrollo: Los recursos minerales en México. Problemas del Desarrollo, Revista Latinoamericana de Economía, México, v.45, n. 179, p. 137-158, octubre-diciembre, 2014.

ASTELARRA, S.; DE LA CAL, V.; DOMÍNGUEZ, D. Conflictos en los Sitios Ramsar de Argentina: aportes para una ecología política de los humedales. Letras Verdes. Revista Latinoamericana de Estudios Socioambientales, Quito, n. 22, p. 228-247, septiembre, 2017.

BAMBER, Alison; HASANALI, Stephanie; NAIR, Anil; WATKINS, Sharon; VIGIL, Daniel; VAN DYKE, Michael; MCMULLIN, Tami et al. A Systematic Review of the Epidemiologic Literature Assessing Health Outcomes in Populations Living near Oil and Natural Gas Operations: Study Quality and Future Recommendations. International journal of environmental research and public health, v. 12, n. 16, jun. 2019. Disponível em: https://doi.org/10.3390/ijerph16122123. Acesso em: 17 oct. 2020.

BJORLYKKE, Knut. Petroleum Geoscience: From Sedimentary Environments to Rock Physics. 2, Oslo: Springer, 2015.

BLACKWILL, Robert D.; O’ SULLIVAN, Megan L. Americas Energy Edge. The Geopolitical Consecuences of the Shale Revolution. Foreign Affairs, Nueva York, marzoabril, 2014. Disponível em: https://www.foreignaffairs.com/articles/united-states/2014-0212/americas-energy-edge . Acesso em: 10 oct. 2020

BROSWIMMER, Franz J. Ecocidio: Breve historia de la extinción en masa de las especies, México: Océano, 2002.

CANTE, Freddy Eduardo. Una política de mínimo extractivismo. Estudios Políticos, Antioquia, p. 129-149, 2015.

CARDOZA, Rubén. Los derechos de los pueblos indígenas y el derecho a un medio ambiente sano: derechos humanos considerados principalmente en la reforma constitucional en materia energética. Anuario de derecho constitucional latinoamericano, Bogotá, Año XXIII, pp. 243$261,2017$.

CASEY, Joan; SAVITZ, David.; RASMUSSEN, Sara; OGBURN, Elizabeth; POLLAK, Jonathan; MERCER, Dione; SCHWARTZ, Brian. Unconventional Natural Gas Development and Birth Outcomes in Pennsylvania, USA. Epidemiology, v.27, n. 2, p. 163-172. 2016. Disponível em: https://doi.org/10.1097/EDE.0000000000000387 . Acesso em: 17 oct. 2020.

CONSEJO NACIONAL DE EVALUACIÓN DE LA POLÍTICA DE DESARROLLO SOCIAL. Estudio diagnóstico del derecho a un medio ambiente sano 2018. Ciudad de México. 2018. 
CHARRY-OCAMPO, Sharel; PEREZ, Aníbal. Efectos de la estimulación hidráulica (fracking) en el recurso hídrico: Implicaciones en el contexto colombiano. Ciencia e Ingeniería Neogranadina, v.28, n. 1, p. 135-164, 2018.

DE LA VEGA, Ángel; RAMÍREZ, Jaime. El gas de lutitas (shale gas) en México. Recursos, explotación, usos, impactos. Economía UNAM, México, v. 12, n. 34, p. 83-84, 2015.

DELGADO, Gian Carlo (Coord.). Ecología política del extractivismo en América Latina: casos de resistencia y justicia socioambiental, Buenos Aires: CLACSO, 2013.

ESTRADA, Javier. Desarrollo del gas de lutita (shale gas) y su impacto en el mercado energético de México: Reflexiones para Centroamérica. México: CEPAL/Sede subregional México, 2013.

FALERO, Alfredo (a). La expansión de la economía de enclaves en América Latina y la ficción del desarrollo: siguiendo una vieja discusión en nuevos moldes. Revista Mexicana de Ciencias Agrícolas, México, v. 1, p. 145-157, 2015.

FALERO, Alfredo (b). La potencialidad heurística del concepto de economía de enclave para repensar el territorio. Revista NERA, Año 18, n. 28, p. 223-240, 2015.

FREIER, Alexander; SCHAJ, Gretel. La fractura hidráulica en Argentina: los cambios en el concepto de territorialidad y la emergencia de nuevos regímenes de soberanía. Revista Enfoques: Ciencia política y administración pública, Santiago, v. XIV, n. 25, p. 59-81, 2016.

GROSFOGUEL, Ramón. Del $<<$ extractivismo económico $>>$ al $<<$ extractivismo epistémico $>>$ y al $<<$ extractivismo ontológico $>>$ : una forma destructiva de conocer, ser y estar en el mundo. Tabula Rasa, Bogotá, n. 24, p. 123-143, enero-junio 2016.

GUDYNAS, Eduardo. Teología de los extractivismos. Tabula Rasa, Bogotá, n. 24, p. 11-23, enero-junio, 2016.

GUDYNAS, Eduardo. Diez tesis urgentes sobre el nuevo extractivismo. Contextos y demandas bajo el progresismo sudamericano actual. En: Eduardo Gudynas, et. al., Extractivismo, politica y sociedad, Quito: Centro Andino de Educación Popular/Centro Latino Americano de Ecología Social, 2009, p. 187-225.

HILL, Elaine. Shale Gas Development and Infant Health: Evidence from Pennsylvania. Journal of Health Economics, n.61, p.134-150, sept., 2018.

JANDA, Karel; KONDRATENKO, Ivan. An Overview of Economic Impacts of U. S. Shale Gas Revolution. Munich Personal RePec Archive, Múnich, Paper No. 83946, p.1-31, enero, 2018.

LEFF, Enrique. Saber ambiental sustentabilidad, racionalidad, complejidad, poder. 2. ed. México: Siglo XXI Editores, 2002.

MANZANARES, José Luis. Uso del agua en la extracción de gas de lutitas en el noreste de México. retos de regulación ambiental. Estudios Sociales, México, v. XXII, n.44, p. 171-197, julio-diciembre, 2014. 
MARTINEZ-ALIER, Joan. The environmentalism of the poor: a study of ecological conflicts and valuation. Massachusetts: Edward Elgar Publishing, 2002.

MARTINEZ-ALIER, Joan. Social Metabolism, Ecological Distribution Conflicts, and Languages of Valuation. Capitalism Nature Socialism, v.20, n.1, p.58-87, 2009. Disponível em: https://doi.org/10.1080/10455750902727378 . Acesso em: 3 oct. 2020

MARTINEZ-ALIER, Joan; KALLIS, Giorgios; VEUTHEY, Sandra; WALTER, Mariana; y, TEMPER, Leah. Social Metabolism, Ecological Distribution Conflicts, and Valuation Languages. Ecological Economics, n.70, p. 153-158, 2010. Disponível em: https://doi.org/10.1080/10455750902727378 . Acesso em: 3 oct. 2020

MCKENZIE, Lisa; GUO, Ruixin; WITTER, Roxana; SAVITZ, David; NEWMAN, Lee; ADGATE, John. Birth outcomes and maternal residential proximity to natural gas development in rural Colorado. Environmental Health Perspectives, v. 122, n. 4, p. 412-417, apr., 2014. Disponível em: https://doi.org/10.1289/ehp.1306722 . Acesso em: 17 oct. 2020

MERCHAND, Marco Antonio. Neoextractivismo y conflictos ambientales en América Latina. Espiral, Guadalajara, v. 23, n. 66, p. 157-159, mayo-agosto, 2016.

MIRÓN, María Dolores. Oikos y oikonomía: El análisis de las unidades domésticas de producción y reproducción en el estudio de la Economía antigua. Gerión. Revista de Historia Antigua, Madrid, v. 22, n. 1, p. 61-79, 2005.

MURILLO, Diana Carolina Murillo; SACHER, William Sacher. Nuevas territorialidades frente a la megaminería: el caso de la Reserva Comunitaria de Junín. Letras Verdes. Revista Latinoamericana de Estudios Socioambientales, Quito, n. 22, p. 46-70, septiembre, 2017.

NACIONES UNIDAS. Declaración Universal de los Derechos Humanos. 1948.

NACIONES UNIDAS. Pacto Internacional de Derechos Económicos, Sociales y Culturales. 1966.

NACIONES UNIDAS. Convención sobre la eliminación de todas las formas de discriminación contra la mujer. 1979.

NACIONES UNIDAS. Observación General 14 del CDESC. 2000.

NY DEPARTAMENT OF HEALTH. A Public Health Review of High Volume Hydraulic Fracturing for Shale Gas Development, Nueva York, diciembre, 2014. Disponível em: https://www.health.ny.gov/press/reports/docs/high_volume_hydraulic_fracturing.pdf NÚNEZ, Cristina. How Has Fracking Changed Our Future? National Geographic, Whashington, 2013. Disponível em: https://n9.cl/3w2b . Acesso em: 10 oct. 2020.

OLIVERA, Beatriz (Coord.). Impactos del fracking en las mujeres, México: Alianza Mexicana contra el fracking/Coordinadora Regional de Acción Solidaria en Defensa de las Huastecas y el Totonacapan (CORASON)/Fundar, Centro de Análisis e Investigación A.C., 2019. 
ORGANIZACIÓN INTERNACIONAL DEL TRABAJO. Convenio 169, 1989. Disponível em: https://n9.cl/03irp . Acesso em: 7 nov. 2020.

ORGANIZACIÓN MUNDIAL DE LA SALUD. Documentos básicos, 2014. Disponível em: https://apps.who.int/gb/bd/PDF/bd48/basic-documents-48th-edition-sp.pdf?ua=1\#page=7 . Acesso em: 7 nov. 2020.

ORGANIZACIÓN MUNDIAL DE LA SALUD. Salud y derechos humanos. Notas Descriptivas, 2017. Disponível em: https://n9.cl/01xl . Acesso em: 7 nov. 2020.

PARRAGUEZ, María Luisa; UGARTE, Alberto; CAMPERO, Georgina. Shale Gas in the United States: Transforming Energy Security in the Twenty-First Century. Norteamérica, México, Año 10, n. 1, p. 7-38, enero-junio, 2015.

PORRIT, Jonathon (Coord.). Salvemos la Tierra, México: Aguilar Editor, 1991.

PROSALUS; CRUZ ROJA ESPAÑOLA. Comprendiendo el Derecho Humano a la Salud. España. 2014.

RASMUSSEN, Sara; OGBURN, Elizabeth; MCCORMACK, Meredith; CASEY, Joan; BANDEEN-ROCHE, Karen; MERCER, Dione; SCHWARTZ, Brian. Asthma Exacerbations and Unconventional Natural Gas Development in the Marcellus Shale. JAMA Intern Med. v. 176, n. 9, p. 1334-1343, Sep., 2016.

RIECHMANN, Jorge. El socialismo puede llegar sólo en bicicleta. Ensayos ecosocialistas, Madrid: Catarata, 2012.

ROJAS-RUEDA, David. Impactos en salud pública del fracking (extracción de gas por medio de fractura hidráulica) en España. Gaceta sanitaria, Barcelona, v. 27, n 4, p. 13-16, julioagosto, 2013.

STACY, Shaina; BRINK, LuAnn; LARKIN, Jacob; SADOVSKY, Yoel; GOLDSTEIN, Bernard; PITT, Bruce; ØTALBOTT, Evelyn. Perinatal Outcomes and Unconventional Natural Gas Operations in Southwest Pennsylvania. PLoS One. n. 10, v.6, e012642, 2015. Disponível em: https://doi.org/10.1371/journal.pone.0126425 . Acesso em: 17 oct. 2020.

TOLEDO, Víctor Manuel. Naturaleza, producción, cultura: Ensayos de ecología política, Xalapa: Universidad Veracruzana, 1989.

TOLEDO, Víctor Manuel. El Metabolismo Social: Una nueva teoría socioecológica. Relaciones, Estudios de Historia y Sociedad, v. 34, n. 136, p. 41-71, nov, 2013.

TETREAULT, Darcy, OCHOA Heriberto y HERNÁNDEZ Eduardo. Conflictos socioambientales y alternativas de la sociedad civil, Guadalajara: ITESO, 2012.

TUNCAK, Bascut. Palabras de apertura del relator especial de las Naciones Unidas sobre derechos humanos y sustancias y desechos peligrosos, $73^{\circ}$ período de sesiones de la Asamblea General de la ONU. 2018. Disponível em: https://n9.cl/3a17 . Acesso em: 7 nov. 2020. 
UNION INTERPARLAMENTARIA. Derechos Humanos. Manual para parlamentarios No. 26. Ginebra, 2016 Disponível em: https://n9.cl/qwj0m . Acesso em: 7 nov. 2020.

U. S. ENERGY INFORMATION ADMINISTRATION. Glossary: Hydraulic fracturing. 2020. Disponível em: https://www.eia.gov/tools/glossary/index.php?id=H . Acesso em: 10 oct. 2020.

VARGAS, Rosío; BARRIOS, Heberto. El impacto geopolítico de la revolución del gas de esquisto: consideraciones para México. El Cotidiano, n.177, p.61-68, enero-febrero, 2013.

VÁZQUEZ, Carlos; OROZCO Alma. La destrucción de la naturaleza, México: FCE, 1995.

Data de recebimento: 10.09.2020

Data de aprovação: 19.02.2021 